\title{
$225 \mathrm{GHz}$ Atmospheric Opacity Measurements from Two Arctic Sites
}

\author{
S. Matsushita ${ }^{1}$, Ming-Tang Chen ${ }^{1}$, P. Martin-Cocher ${ }^{1}$, K. Asada ${ }^{1}$, \\ C.-P. Chen ${ }^{1}$, M. Inoue ${ }^{1}$, S. Paine ${ }^{2}$, D. Turner ${ }^{3}$ and E. Steinbring ${ }^{4}$ \\ ${ }^{1}$ Institute of Astronomy and Astrophysics, Academia Sinica, \\ P.O.Box 23-141, Taipei 10617, Taiwan, R.O.C. \\ email: satoki@asiaa.sinica.edu.tw \\ ${ }^{2}$ Smithsonian Astrophysical Observatory, \\ 160 Concord Ave., Cambridge, MA 02138, USA \\ ${ }^{3}$ National Severe Storms Laboratory, \\ 120 David L. Boren Boulevard, Norman, OK, 73072, USA \\ ${ }^{4}$ National Research Council, Herzberg Inst of Astrophysics, \\ 5071 W Saanich Rd, CA Victoria BC V9E 2E7, Canada
}

\begin{abstract}
We report the latest results of $225 \mathrm{GHz}$ atmospheric opacity measurements from two Arctic sites; one on high coastal terrain near the Eureka weather station, on Ellesmere Island, Canada, and the other at the Summit Station near the peak of the Greenland icecap. This is a campaign to search for a site to deploy a new telescope for submillimeter Very Long Baseline Interferometry and $\mathrm{THz}$ astronomy in the northern hemisphere. Since 2011, we have obtained 3 months of winter data near Eureka, and about one year of data at Summit Station. The results indicate that these sites offer a highly transparent atmosphere for observations in submillimeter wavelengths. Summit Station is particularly excellent, and its zenith opacity at $225 \mathrm{GHz}$ is statistically similar to the Atacama Large Millimeter/submillimeter Array site in Chile. In winter, the opacity at Summit Station is even comparable to that observed at the South Pole.
\end{abstract}

Keywords. Arctic sites, $225 \mathrm{GHz}$ opacity, site testing

\section{Introduction}

The success of Doeleman et al. (2008) in obtaining a scatter-free size estimate of submillimeter emission in Sagittarius A* (Sgr A*) using Very Long Baseline Interferometry (VLBI) promises a new window for direct imaging of supermassive black holes (SMBHs). Although Sgr $\mathrm{A}^{*}$ is the nearest known and biggest (in apparent size), its mass is relatively small among SMBH. This means a short timescale of variability, leading to undesirable smoothing of that signal during integration. On the other hand, the second largest source in apparent size is the SMBH in M87 (Virgo A), which has a large mass. A further scientifically interesting point is that M87 has a strong jet activity. But to image the SMBH in M87 with submm-VLBI requires a longer baseline in the northern hemisphere than currently available (see Inoue et al. 2012 in this proceeding) and so we began a search for a new submm telescope site.

\section{Site Selection}

For the site selection, we set criteria as follows: (1) annual precipitable water vapor (PWV) of less than $3 \mathrm{~mm}$ for good submm opacity; (2) longest-possible baseline with existing telescopes, for best imaging resolution; (3) observable sky together with the 

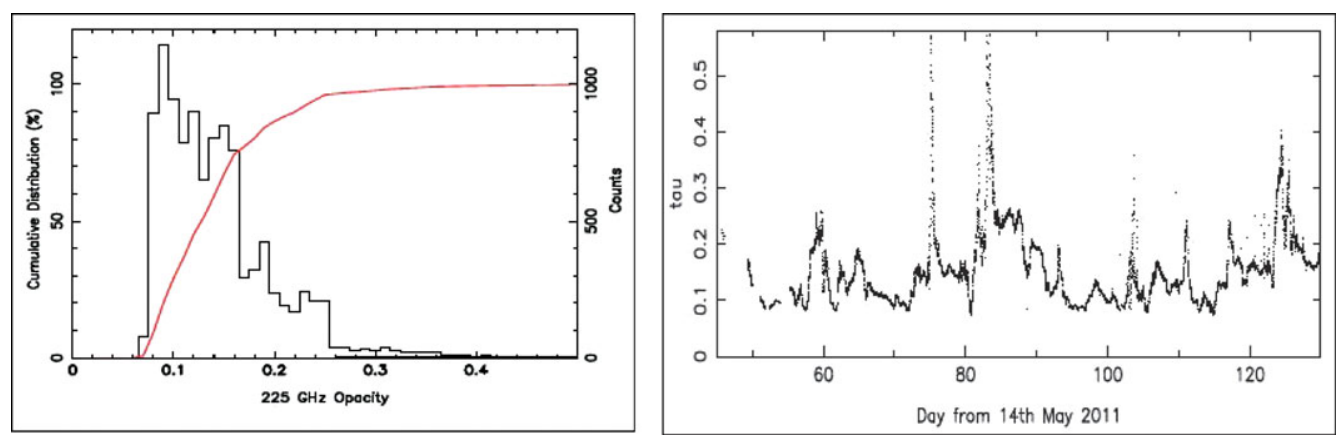

Figure 1. (Left) Time variation plot of $225 \mathrm{GHz}$ opacity at the PEARL site. (Right) Histogram and cumulative plot of the opacity.

Atacama Large Millimeter/submillimeter Array (ALMA) to achieve the highest possible sensitivity; (4) accessibility to the site.

Based on the first two criteria, there are three potential broad regions of interest; western China and Tibet, the highest mountains of southern Alaska, or the high Arctic polar desert, including northern Canada and Greenland. The Western China and Tibet region does not have common sky with ALMA, so it does not meet criterion (3), and the tallest peaks in Alaska (e.g., Mount McKinley) are excluded due to criterion (4). The Eureka research base on Ellesmere Island, Canada and Summit on the Greenland icecap meet all four criteria, and we considered these two sites for further study.

\section{3. $225 \mathrm{GHz}$ Tipping Radiometer}

For the site survey, we purchased a $225 \mathrm{GHz}$ tipping radiometer from Radiometer Physics GmbH. The reason for the choice of this frequency is that there are many site survey results from all over the world, including the summit of Mauna Kea, the ALMA (Chajnantor) site and South Pole.

For the opacity measurements, we use the tipping method: We observe five angles $\left(90^{\circ}, 42^{\circ}, 30^{\circ}, 24^{\circ}\right.$, and $19.2^{\circ}$, corresponding to $\sec (z)$ of $1.0,1.5,2.0,2.5$, and 3.0$)$ with 4 second integration at each angle, for a duration of 75 second per tipping measurement. For each measurement, the opacity is derived from the instrument output voltage as a function of zenith angle. Measurements were obtained every 10 minutes.

We first tested at the Academia Sinica, Institute of Astronomy and Astrophysics (ASIAA) in Taipei, Taiwan, and then repeated on Mauna Kea, Hawaii to check the consistency of our measurements with the $225 \mathrm{GHz}$ tipping radiometer at the Caltech Submillimeter Observatory (CSO). Simultaneous opacity measurements were performed between 31 December 2010 and 11 January 2011, and are consistent with each other (linear regression coefficient $=1.04$ ).

\section{Results}

Identical observations were then carried out at the high Arctic sites, allowing direct comparison to those worldwide.

\subsection{Eureka, Ellesmere Island, Canada}

Eureka is a manned weather station on Ellesmere Island, Canada. It has a 5000-foot all-season airstrip. Yearly resupply by ship occurs in late summer. A road allows access to the Polar Environment Atmospheric Research Laboratory (PEARL), located on a $610 \mathrm{~m}$-high ridge at $80.05^{\circ} \mathrm{N}, 86.42^{\circ} \mathrm{W}$. Although this site is not as high as other good 

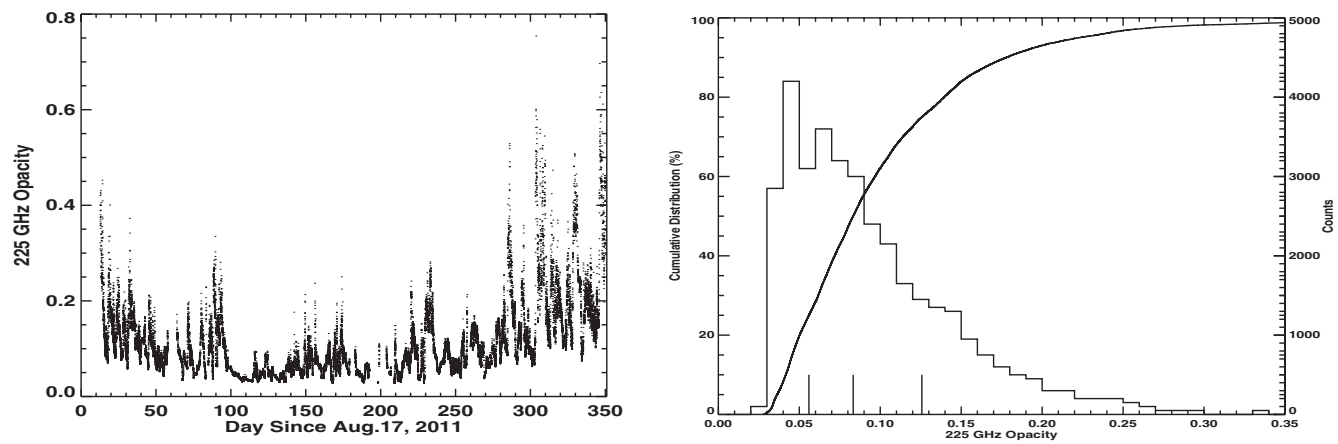

Figure 2. (Left) Time variation plot of $225 \mathrm{GHz}$ opacity at the Summit Camp. (Right) Histogram and cumulative plot of the opacity.

submm sites, it is expected to have low opacity conditions due to the low temperatures, typically between $-20^{\circ} \mathrm{C}$ and $-40^{\circ} \mathrm{C}$ in winter (Steinbring et al. 2010). The radiometer was deployed on the rooftop observing platform of PEARL, and measured the $225 \mathrm{GHz}$ opacity between 14 February and 10 May 2011. Tipping direction was south, providing 10,522 data points.

Fig. 1 shows the time variation, histogram, and cumulative plots. The lowest $225 \mathrm{GHz}$ opacity measured was 0.07 , the $25 \%$ quartile was 0.11 , the median ( $50 \%$ quartile) was 0.14 , and the most frequent opacity was 0.09 during our measurements. These statistics indicate that submm-VLBI at this frequency is feasible at this site.

\subsection{Summit Camp, Greenland}

Summit Camp is a research station near the peak of the Greenland ice sheet, located at $72.57^{\circ} \mathrm{N}, 38.46^{\circ} \mathrm{W}$, with an elevation of $3,200 \mathrm{~m}$. Access is by C-130 air transport in summer and by Twin Otter in winter, or traverse from Thule. Very good opacity conditions are expected for this site, based on the low winter temperatures, between $-40^{\circ} \mathrm{C}$ and $-60^{\circ} \mathrm{C}$ with minimum temperature of $-72^{\circ} \mathrm{C}$ (Vaarby-Laursen 2010), and the high altitude. We put our radiometer on the roof of the Mobile Science Facility (MSF), and started measuring the opacity on 17 August 2011 (and still continue to do so). Here we show data up to 31 July 2012. Tipping directions were both south and north, and provide 36,555 data points.

Fig. 2 shows the time variation, histogram, and cumulative plots. The lowest $225 \mathrm{GHz}$ opacity measured was 0.027 , the $25 \%$ quartile was 0.056 , the median was 0.083 , and the most frequent opacity was 0.04 during our measurements. These statistics are excellent, strongly indicating that submm-VLBI at these frequencies, or even higher, is feasible at this site. $\mathrm{THz}$ astronomy is also worth considering.

\subsection{Comparison with Other Sites}

To illustrate how a high Arctic submm site can compare to the best worldwide, we compared Summit Camp statistics with those of the ALMA site (elevation $=5,050 \mathrm{~m}$ ) and South Pole $(2,800 \mathrm{~m})$. The opacity data for the ALMA site have been taken from Radford \& Chamberlin (2000) and Radford (2011), measured between April 1995 and April 2006, and the data for South Pole from Chamberlin \& Bally (1994) and Chamberlin \& Bally (1995), measured between January and December 1992.

Winter in the northern hemisphere is defined as between the beginning of November and the end of April, with summer May through October. The opposite is taken to be the case for the southern hemisphere; winter May through October, summer November 

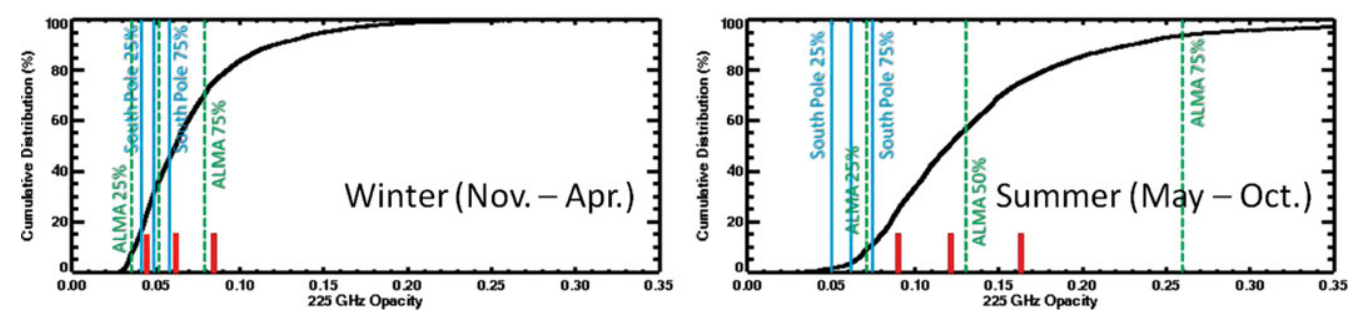

Figure 3. Opacity quartiles comparison between the Summit Camp (histogram and cumulative plots with quartiles as short and thick solid lines), the ALMA site (dashed vertical lines), and South Pole (thin solid vertical lines). Left plots are winter season, and right summer.

through April. We put the quartiles for the three sites on the cumulative plots of the Summit Camp in Fig. 3.

In winter season, $225 \mathrm{GHz}$ opacities of all three sites are less than 0.1 even at the $75 \%$ quartile. There is small difference between the sites, but the opacity statistics of Summit Camp are rather similar to that of the ALMA site. The opacity statistics of South Pole do not vary much from winter to summer, but the ALMA site shows much greater variation. Summit Camp falls somewhere in between. It is worth noting, however, that 2011/12 represents a record warm period for Greenland, which may have affected opacity statistics. Long-term monitoring is needed to carefully judge the quality of Summit Camp as a submm site.

\section{Summary}

We have presented a program of site testing for a submm telescope site in the northern hemisphere. Both Eureka, Ellesmere Island, and Summit Camp, Greenland offer the potential for new submm VLBI observations. Based on the best $225 \mathrm{GHz}$ opacity measurements, we selected Summit Camp, and efforts are currently underway to retrofit an antenna for the extremes of the site. Opacity measurements are still ongoing to collect long-term opacity variation data. This will reveal whether the record warmth in Greenland has affected the opacity statistics or not, and the true fraction of time that Summit Camp reaches the quality of South Pole. In addition, atmospheric characterisation using various instrumentations (cloud radars and lidars, radiosondes, microwave radiometers, precipitation measurements) are ongoing at Summit Camp by atmospheric researchers (e.g., Shupe et al. 2012). We are closely collaborating with them to estimate the atmospheric conditions more accurately, and to construct accurate atmospheric models for future submm/THz astronomy at this site.

\section{References}

Chamberlin, R. A. \& Bally, J. 1994, App. Opt. 33, 1095

Chamberlin, R. A. \& Bally, J. 1995, Int. J. IR MM Waves 16, 907

Doeleman, S. S., et al. 2008, Nature 455, 78

Inoue, M., et al. 2012, these Proceedings

Radford, S. J. E. 2011, RevMexAA (SC) 41, 87

Radford, S. J. E. \& Chamberlin, R. A. 2000, ALMA Memo 334

Shupe, M. D., et al. 2012, Bull. Amer. Meteo. Soc. in press

Steinbring, E., Carlberg, R., Croll, B., Fahlman, G., Hickson, P., Ivanescu, L., Leckie, B., Pfrommer, T., \& Schoeck, M. 2010, PASP 122, 1092

Vaarby-Laursen, E., DMI Tech. Rep. 10-09 